\title{
Corrigendum to "A Generating Function Approach to HIV Transmission with Dynamic Contact Rates"
}

\author{
E.O. Romero-Severson ${ }^{1 *}$, G.D. Meadors ${ }^{2}$, E.M. Volz ${ }^{3}$ \\ ${ }^{1}$ Theoretical Biology and Biophysics, Los Alamos National Laboratory, Los Alamos, NM \\ ${ }^{2}$ Department of Physics, University of Michigan, Ann Arbor, MI \\ ${ }^{3}$ Department of Epidemiology, University of Michigan, Ann Arbor, MI
}

\section{Corrections}

We found a few issues in our original paper that require correction. First, we did not state a basic assumption used to derive an expression for $R_{*}$. In our infection model we included the term $\omega$ to account for the rate of 'natural removal' from the population (i.e. cessation of sexual activity that is not related to HIV-specific mortality). We did not clearly state that our derivations assume that $\omega=0$. This assumption was justified by the fact that $\delta_{1}$ is always going to be much larger than $\omega$ for HIV such that the probability of an infected case being removed before reaching the second stage of infection is very small. For example, if we assume that the acute stage of infection lasts 2 months and that an average person is sexually active for 30 years, then the probability of being removed before progressing to the second stage is less than 0.005 . We chose to assume $\omega=0$ but did not clearly state this assumption.

Second, there is a typo in the original expression for $R_{*}$ where the terms $\beta_{1}$ and $\beta_{2}$ were omitted. The correct expression for $R_{*}$ is

$$
\begin{aligned}
R_{*} & =\frac{\rho}{\lambda_{1}}\left[\widetilde{c}_{1}(1)+i_{1}^{\prime}(1) c_{1}^{\prime}(1)+c_{s}^{\prime}(1)+i_{2}^{\prime}(1) c_{2}^{\prime}(1)\right]+ \\
& \left(1-\frac{\rho}{\lambda_{1}}\right)\left[\widetilde{c}_{s}(1)+i_{2}^{\prime}(1) c_{2}^{\prime}(1)\right], \\
& =\frac{\rho}{\lambda_{1}}\left[\frac{(k+1) \theta \beta_{1}}{\lambda_{1}}+\frac{1-\phi_{1}}{\phi_{1}} \frac{k \theta \beta_{1}}{\lambda_{1}}+k \theta\left[\frac{\beta_{1}}{\lambda_{1}}+\frac{\beta_{2}}{\lambda_{2}}\right]+\frac{1-\phi_{2}}{\phi_{2}} \frac{k \theta \beta_{2}}{\lambda_{2}}\right]+ \\
& \left(1-\frac{\rho}{\lambda_{1}}\right)\left[(k+1) \theta\left[\frac{\beta_{1}}{\lambda_{1}}+\frac{\beta_{2}}{\lambda_{2}}\right]+\frac{1-\phi_{2}}{\phi_{2}} \frac{k \theta \beta_{2}}{\lambda_{2}}\right] .
\end{aligned}
$$

Second, there was an error in the plotting code for figures 2, 3 and 4 . In the case of figures 2 and 3 we neglected to set $\omega=0$ in the plotting code leading to an imperceptibly small error in the figures. However, in the case of figure 4 we used an incorrectly defined Gamma random variable that significantly altered the appearance of the figure. The correct figures are given below

*Corresponding author. E-mail: eoromero@lanl.gov 

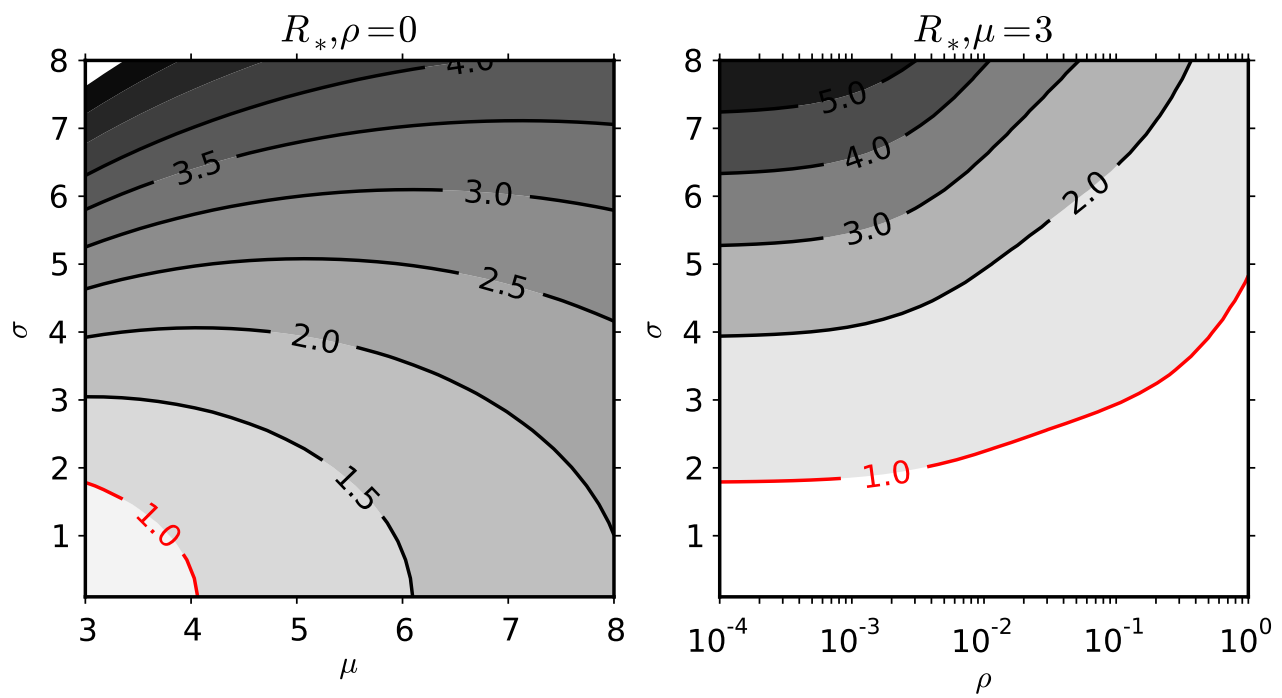

FiguRE 1. Effect of contract rate volatility on $R_{*}$. We display (left panel) the effect of the mean (x-axis) and standard deviation (y-axis) of the contact rate distribution on the basic reproduction number without contact rate volatility. The panel on the right shows the effect of the standard deviation of contact rates (y-axis) and the degree of contact rate volatility (x-axis) on the basic reproduction number. The average duration of a behavioral interval is $\rho^{-1}$ such that low values of $\rho$ signify essentially-static contact rates. The average duration of infection is 148 months with the first 2 months being the first stage and the remainder in the second. The probabilities of infection given contact are 0.05 and 0.001 in the first and second stages respectively. 

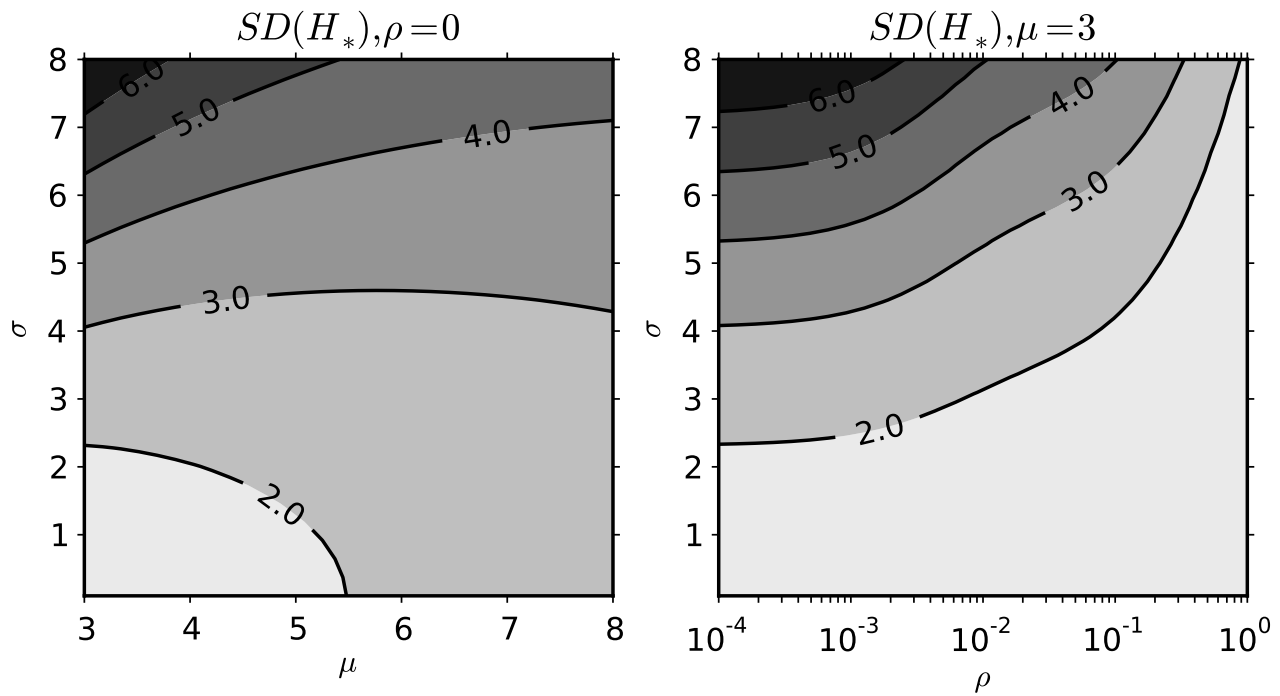

FIgURE 2. Effect of contract rate volatility on $S D\left(H_{*}\right)$. This plot shows the effect of the mean (x-axis) and standard deviation (y-axis) of the contact rate distribution on the standard deviation of the number of transmissions generated by an index case, $S D\left(H_{*}\right)$, without contact rate volatility (left panel). The panel on the right shows the effect of the standard deviation of contact rates (y-axis) and the degree of contact rate volatility (x-axis). The average duration of a behavioral interval is $\rho^{-1}$ such that low values of $\rho$ mean essentially static contact rates. The average duration of infection is 148 months with the first 2 months being the first stage and the remainder in the second. The probabilities of infection given contact are 0.05 and 0.001 in the first and second stages respectively. 

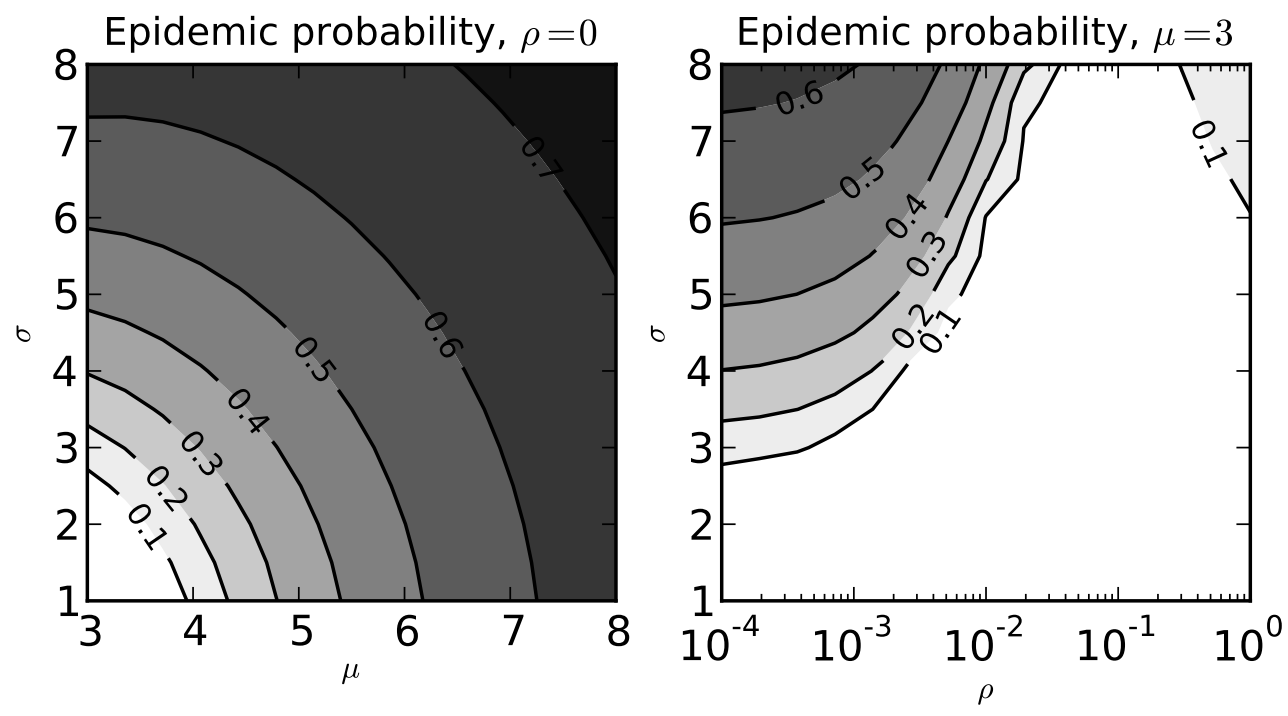

FIgURE 3. Effect of contract rate volatility on the probability of an epidemic. This plot shows the effect of the mean (x-axis) and standard deviation (y-axis) of the contact rate distribution on the probability of an epidemic without contact rate volatility (left panel). The panel on the right shows the effect of the standard deviation of contact rates (y-axis) and the degree of contact rate volatility (x-axis). The average duration of a behavioral interval is $\rho^{-1}$ such that low values of $\rho$ mean essentially static contact rates. The average duration of infection is 148 months with the first 2 months being the first stage and the remainder in the second. The probabilities of infection given contact are 0.05 and 0.001 in the first and second stages respectively. 